\title{
ON SUBDIRECT PRODUCTS OF RINGS WITHOUT SYMMETRIC DIVISORS OF ZERO
}

\author{
TAO-CHENG YIT ${ }^{1}$
}

ABSTRACT. A theorem of V. A. Andrunakievič and Ju. M. Rjabuhin asserts that a ring $R$ is without nilpotent elements if and only if $R$ is a subdirect product of skew-domains. In this paper we prove that a semiprime ring $R$ with involution is a subdirect product of rings without symmetric divisors of zero if and only if $R$ is compressible for its symmetric elements.

Introduction. Let $R$ be a ring with involution *, $S$ the set of all symmetric elements $\left(x=x^{*}\right)$ of $R, T$ the set of all traces $\left(t+t^{*}\right)$ of $R, N$ the set of all norms $\left(t t^{*}\right)$ of $R$, and $S_{0}=T \cup N \subseteq S$. Given a symmetric ideal $I=I^{*}$ of $R$, the factor ring $R / I$ will be equipped with the canonical invo-

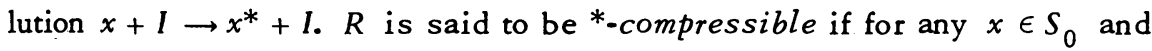
$n$ equal to a power of $2, a x^{n} b=0$ implies $a x b=0$.

Andrunakievič and $\mathrm{Rj}$ abuhin have shown that a ring $R$ is without nilpotents if and only if $R$ is a subdirect product of skew-domains [1]. Related to this, one would like to ask when $R$ has a subdirect representation into rings without symmetric divisors of zero. We prove here that a semiprime ring $R$ is *-compressible if and only if $R$ is a subdirect product of rings without symmetric divisors of zero (in the case of 2-torsion free).

Remark 1. Let $R=F_{2}$ be the ring of matrices over a field $F$. Assume that for a given involution*, the symmetric divisors of zero are zero alone. Then * must be the symplectic involution (i.e., $\left.\left(\begin{array}{ll}a & b \\ c & d\end{array}\right)^{*}=\left(\begin{array}{cc}d & -b \\ -c & a\end{array}\right)\right)$. In fact if * were not the symplectic involution then, by a result of Jacobson [7, Case A, P.311], * must be of the following type:

Presented to the Society, February 19, 1973; received by the editors September 7, 1973.

AMS (MOS) subject classifications (1970). Primary 16A28; Secondary 16A48.

Key words and phrases. Ring with involution, symmetric elements, traces, norms, *-prime rings, $m$-system, *-compressible rings.

1 This research was supported partly by Ontario Graduate Fellowship and partly by Department of Mathematics, Carleton University, Ottawa, Canada. 


$$
\left(\begin{array}{ll}
a & b \\
c & d
\end{array}\right)^{*}=\left(\begin{array}{cc}
r_{1}^{-1} \bar{a} r_{1} & r_{1}^{-1} \bar{c} r_{2} \\
r_{2}^{-1} \bar{b} r_{1} & r_{2}^{-1} \bar{d} r_{2}
\end{array}\right)
$$

where $F$ has an involution $\lambda \rightarrow \bar{\lambda}$ and $\bar{r}_{i}=r_{i}(i=1,2)$ are fixed invertible symmetric elements of $F$. Now take $x=\left(\begin{array}{cc}0 & 0 \\ 1 & 0\end{array}\right)$; then $x^{2}=0$. Then

$$
x x^{*}=\left(\begin{array}{cc}
0 & 0 \\
0 & r_{1}^{-1} r_{2}
\end{array}\right) \neq 0 \text { and } x\left(x x^{*}\right)=0 \text {. }
$$

This means $x x^{*}$ is a symmetric divisor of zero, a contradiction. This shows that $*$ must be the symplectic one.

Remark 2. C. Lanski has characterized a semiprime ring $R$ such that all its nonzero symmetric elements do not annihilate themselves in the case of 2-torsion free; namely, that $R$ has one of the following types: (i) a skewdomain, (ii) a subring of the direct sum of a skew-domain and its opposite with interchanging co-ordinate involution, or (iii) an order in $2 \times 2$ matrices over a field [9]. Montgomery and Herstein extended Lanski's characterization to the case where $R$ is any semiprime ring with involution such that all its nonzero traces do not annihilate themselves. In case (i), $R$ is, of course, *-compressible. In case (ii), $R$ is a subring of the direct sum of *-compressible rings, and, consequently, $R$ is again *-compressible. In case (iii), by using Remark 1 we get $S_{0} \subseteq Z\left(F_{2}\right)$, the center of $F_{2}$, and so $R$ is *compressible. This shows that any semiprime ring with involution such that all its nonzero traces do not annihilate themselves is a *-compressible ring.

We first prove several propositions.

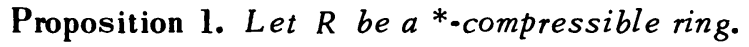

(1) If $s \in S_{0}$ with $s^{n}=0$, then $s=0$.

(2) If $x x^{*}=0$ then $x^{*} x=0$.

Proof. (1) follows immediately from the definition of a $*_{\text {-compressible }}$ ring. As for (2), note that $\left(x^{*} x\right)^{2}=0$ and apply (1).

Recall that a ring $R$ is *-prime if for any symmetric ideals $A=A^{*}$ and $B=B^{*}$ of $R, A B=0$ implies $A=0$ or $B=0$.

Proposition 2. Let $R$ be $a^{*}$-prime ring. Then $R$ is *-compressible if and only if every nonzero symemtric element in $S_{0}$ is a nondivisor of zero.

Proof. "If" part. This is an immediate consequence of Remark 2. "Only if" part. First we show that $s d=0$ with $s, d \in S_{0}$ implies $s=0$ or $d=0$. 
Let $x \in R$. We have

$$
\begin{aligned}
s\left(s x d+d x{ }^{*} s\right)^{2} d & \\
& =s\left(s x d s x d+d x{ }^{*} s^{2} x d+s x d^{2} x * s+d x * s d x * s\right) d=0
\end{aligned}
$$

(because $d s=s d=0$ ).

By the hypothesis, $s\left(s x d+d x^{*} s\right) d=0$. This means that $s^{2} x d^{2}=0 \forall x \in R$. Since $R$ is *-prime, $s^{2}=0$ or $d^{2}=0$. Since $R$ is *-compressible, $s=0$ or $d=0$. By Lanski's characterization, mentioned in Remark $2, R$ is without nonzero symmetric divisors of zero in $S_{0}$.

Recall that a subset $M_{0}$ of a ring $R$ is an m-system if $a, b \in M_{0}$ imply $a x b \in M_{0}$ for some $x \in R$.

Proposition 3. Let $R$ be $a^{*}$-compressible ring. Let $M_{0}$ be an m-system excluding (0). Then (0) can be enlarged to a ${ }^{*}$-prime ideal $P$ such that $R / P$ is * compressible and $P \cap M_{0}=\varnothing$.

Proof. Let $M$ be an $m$-system containing $M_{0}$ and maximal with respect to the exclusion of the ideal (0). As is well known, if $J$ is the complement of $M, J=C(M)$, then $J$ is a prime ideal of $R$ excluding $M_{0}$. Assuming for the moment that $J$ has the following property:

for any $s \in S_{0}$ and $x s^{n} y \in J$ which imply $x s y \in J$, where $n$ is a

(1) power of 2 , we take $P=J \cap J^{*}$, and so have a ${ }^{*}$-prime ideal $P$

such that $R / P$ is *-compressible and $P \cap M_{0}=\varnothing$.

It remains to show that $J$ has property (1). Define

$$
\begin{aligned}
M_{1} & =M \cup\left\{x s^{n} y \mid x s y \in M ; n=1,2, \cdots, 2^{h}, \cdots\right\}, \\
M_{2} & =M_{1} \cup\left\{x_{1} s_{1}^{n} y_{1} \mid x_{1} s_{1} y_{1} \in M_{1} ; n=1,2, \cdots, 2^{h}, \cdots\right\}, \\
M_{k+1} & =M_{k} \cup\left\{x_{k} s_{k}^{n} y_{k} \mid x_{k} s_{k} y_{k} \in M_{k} ; n=1,2, \cdots, 2^{h}, \cdots\right\} .
\end{aligned}
$$

Let $M^{\prime}=\bigcup_{k} M_{k}$. Of course, $M^{\prime} \supseteq M$. Now $M^{\prime}$ excludes $(0)$, for if $M_{k}$ excludes $(0)$, $M_{k+1}$ will exclude (0)( since $R$ is *-compressible) and, by construction, $M$ excludes (0). By induction on $k$, all $M_{k}$ exclude (0) and consequently $M^{\prime}$ excludes (0).

Next we show that $M^{\prime}$ is an $m$-system. In fact, it is enough to show that for any pair $a, b \in M_{k}$ there is $c \in R$ such that $a c b \in M^{\prime}$ (for $M \subseteq M_{1} \subseteq$ $\left.M_{2} \subseteq \cdots\right)$. The property holds for $k=1$. Now suppose it is true for $k$; let us show this for $k+1$. Let $a, b \in M_{b+1}$. We wish to find a $c \in R$ such that $a c b \in M^{\prime}$. There are three cases: 
Case 1. Both $a, b \in M_{k}$. Here, $c$ follows from the induction step.

Case 2. Both $a, b \in\left\{x s^{n} y \mid x s y \in M_{k}\right\}$. Here $a=x s^{n} y, b=z d^{m} t$ for some $x, y, t$ and $z$ such that $x s y$ and $z d t \in M_{k}$. By the induction hypothesis, there exists $c \in R$ such that $(x s y) c(z d t) \in M_{k^{\prime}}$ for some $k^{\prime}$. Then $x s^{n}(y c z d t) \in M_{k^{\prime}+1}$ and $\left(x s^{n} y c z\right) d^{m} t \in M_{k^{\prime}+2^{*}}$. Hence $a c b \in M^{\prime}$.

Case 3. If $a \in M_{k}$ and $b \in\left\{x s^{n} y \mid x s y \in M_{k}\right\}$, then we have $a \in M_{k}$ and $b=x s^{n} y$ where $x s y \in M_{k}$. Thus there exists $c \in R$ such that $a c(x s y) \in M_{k^{\prime}}$, for some $k^{\prime}$. So $(a c x) s^{n} y \in M_{k^{\prime}+1}$. Hence $a c b \in M^{\prime}$.

This shows that $M^{\prime}$ is an $m$-system containing $M$. By the above, $M^{\prime}$ excludes (0). By maximality, $M^{\prime}=M$ and so $J=C(M)$ has property (1). The proof is now complete.

We are now in a position to prove the following key theorem.

Theorem 1. Let $R$ be *-compressible, and $\eta$ the prime radical of $R$. Then $\eta=\bigcap\left\{P^{\prime} \mid P^{\prime}\right.$ is $a^{*}$-prime ideal of $R$ and $R / P^{\prime}$ has no nonzero symmetric divisors of zero in $\left.S_{0}\right\}$.

Proof. If $P$ is a prime ideal of $R$, then $M=C(P)$ is an $m$-system excluding (0). By Proposition 3, the ideal (0) can be enlarged to a $*$-prime ideal $P^{\prime}$ such that $R / P^{\prime}$ is *-compressible and $P^{\prime} \cap M=\varnothing$. Thus for such a *-prime ideal $P^{\prime}$, we have $P^{\prime} \subseteq C(M)=P$ and $R / P^{\prime}$ has no nonzero symmetric divisors of zero in $S_{0}$ (Proposition 2). Hence $\eta \supseteq \bigcap\left\{P^{\prime} \mid P^{\prime}\right.$ is a *prime ideal of $R$ and $R / P^{\prime}$ has no nonzero symmetric divisors of zero in $S_{0}$ \}. Since $\eta$ is the least semiprime ideal of $R$, we have the equality; hence the proof is complete.

We can now derive our main theorem.

Theorem 2. Let $R$ be a semiprime ring with involution. Then $R$ is *-compressible if and only if $R$ is a subdirect product of rings without nonzero symmetric divisors of zero in $S_{0}$.

Proof. "Only if" part. It follows immediately from Theorem 1. "If" part. Let $R$ be a subdirect product of rings without symmetric divisors of zero in $S_{0}$. Let $x, y \in R$ and $s \in S_{0}$ with $x s^{n} y=0$. We prove that $x s y=0$. In fact, in each factor $R_{i}$, we have $x_{i} s_{i}^{n} y_{i}=0$. Since $R_{i}$ is semiprime and without symmetric divisors of zero in $S_{0}, R_{i}$ is *-compressible (Remark 2). Thus $x_{i} s_{i} y_{i}=0$ for all indices $i$. Therefore $x s y=0$.

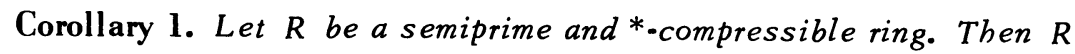
is a subring of a direct product of rings which are either skew-domains or orders in $2 \times 2$ matrices over a field. 
Corollary 2. A semiprime ring $R$ is *-compressible if and only if $x s^{n} y=0$ implies $x s y=0$ for any $s \in S_{0}$ and any integer $n>1$.

Remark 3. If $R$ is 2-torsion free, then we observe that if $S_{0}$ does not contain nonzero divisors of zero, then $S$ itself does not contain nonzero divisors of zero. Also, if $M$ is an $m$-system excluding (0) then $M^{\prime}=$ $\left\{2^{k} x \mid x \in M, k=1,2, \ldots\right\}$ is al so an $m$-system excluding (0). For any *prime ideal $P$ with $P \cap M^{\prime}=\varnothing, R / P$ is 2-torsion free (for if $2 x \in P$ with $x \notin P$ then $2 R\left(x, x^{*}\right) \subseteq P$; since $P$ is *-prime we have $2 R \subseteq P$, a contradiction).

Combining Remark 3 with Proposition 2 and Theorem 1, we have

Theorem 3. Let $R$ be a 2-torsion free and semiprime ring. Then $R$ is *-compressible if and only if $R$ is a subdirect product of rings without nonzero symmetric divisors of zero.

From Theorem 2 we derive the following theorems which generalize $[8],[12]$.

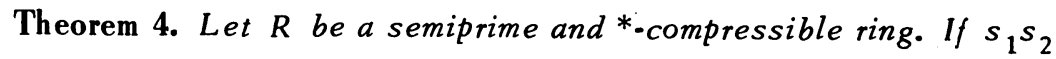
$\cdots s_{n}=0$ with $s_{i} \in S_{0}$, then for any permutation $i_{1}, i_{2}, \cdots, i_{n}$ in the $i$ 's $s_{i_{1}} s_{i_{2}} \cdots s_{i_{n}}=0$ (that is, the product of the $s_{i}$ 's is zero in any order).

Proof. Let $\left({ }_{\lambda} s_{i}\right){ }_{\lambda \in I}$ be the image of $s_{i}$ under a subdirect representation as in Theorem 2. If $s_{1} s_{2} \cdots s_{n}=0$, then ${ }_{\lambda} s_{1} s_{2} \cdots \mu_{\lambda} s_{n}=0$ for all $\lambda \in I$. Using the regularity condition in each factor ${ }_{\lambda} R$ of $R$, there must be $\lambda^{s}{ }_{i}=0$. Thus $\lambda^{s}{ }_{i_{1}} \lambda^{s} i_{2} \cdots \lambda^{s}{ }_{i_{n}}=0$ for all $\lambda \in I$. Consequently $s_{i_{1}} s_{i_{2}} \cdots$ $s_{i_{n}}=0$.

Remark 4. Let $R$ be as in Theorem 4. By using Theorem 2 and Lanksi's theorem, one can see that if $s x d y t=0$ with $s, d, t \in S_{0}$ and $x, y \in R$, then $s d t x y=0$.

$R$ is said to be *-von Neumann regular (*-regular) if for any $s \in S_{0}$ there is $x \in R$ such that $a=a x a$.

Theorem 5. Let $R$ be as in Theorem 4. Assume that $R / P$ is *-regular for every *-prime ideal $P$. Then $R$ is *-regular.

Proof. Let $a \in S_{0}$ and $E$ be the set of all elements of the form

$$
\left(a-a x_{1} a\right)\left(a-a x_{2} a\right) \cdots\left(a-a x_{n} a\right) \text {; }
$$

$x_{1}, x_{2}, \cdots, x_{n}$ running over $R$. Clearly $E$ is closed under multiplication. Then $E$ is an $m$-system. We claim that $0 \in E$. For if $0 \notin E$, then by Prop- 
osition 3, the ideal (0) can be enlarged to a *-prime ideal $P$ such that $R / P$ is *-compressible and $P \cap E=\varnothing$. By the hypothesis, $R / P$ is a *-regular ring and consequently $a-a y a \in P$ for a suitable $y$. But $a-a y a \in E$, a contradiction. We must conclude that $0 \in E$, that is, for some $x_{i} \in R$,

$$
a\left(1-x_{1} a\right) a\left(1-x_{2} a\right) \cdots a\left(1-x_{n} a\right)=0 .
$$

By Remark 4, we have

$$
a^{n}\left(1-x_{1} a\right) \cdots\left(1-x_{n} a\right)=0 .
$$

Thus $a\left(1-x_{1} a\right) \cdots\left(1-x_{n} a\right)=0$. Since the product $\left(1-x_{1} a\right) \cdot\left(\cdot\left(1-x_{n} a\right)\right.$ has the form $1-z a, a(1-z a)=0$, that is, $a=a z a$.

Remark 5. A long standing conjecture of I. Kaplansky [8] was that a ring $R$ is von Neumann regular iff $R$ is a semiprime ring such that each prime image of $R$ is von Neumann regular. This conjecture was settled in the affirmative by I. N. Herstein and E. T. Wong separately in the case of rings without nilpotent elements [12] (see also Fisher and Snider [3]). Theorem 5, whose proof follows the pattem of Herstein-Kaplansky's proof (see [8]), is a generalization of Wong's result.

Acknowledgements. This paper is a part of the author's doctoral dissertation at Carleton University. The author gratefully acknowledges Professor Maurice Chacron for his advice and assistance.

\section{REFERENCES}

1. V. A. Andrunakievič and Ju. M. Rjabuhin, Rings without nilpotent elements, and completely prime ideals, Dokl. Akad. Nauk SSSR 180 (1968), 9-11 = Soviet Math. Dokl. 9 (1968), 565-568. MR 37 \#6320.

2. J. Chacron and M. Chacron, Rings with involution all of whose symmetric elements are nilpotent or regular, Proc. Amer. Math. Soc. 39 (1973), 397-402.

3. J. W. Fisher and R. L. Snider, On the von Neumann regularity of rings with regular prime factor rings (to appear).

4. I. N. Herstein, Noncommutative rings, Carus Math. Monographs, no. 15, Math. Assoc. Amer; distributed by Wiley, New York, 1968. MR 37 \#2790.

5. - Topics in ring theory, Univ. of Chicago Press, Chicago, Ill., 1969. MR $42 \# 6018$.

6. I. N. Herstein and S. Montgomery, Invertible and regular elements in rings with involution, J. Algebra (to appear).

7. N. Jacobson and C. E. Rickart, Homomorphisms of Jordan rings of selfadjoint elements, Trans. Amer. Math. Soc. 72 (1952), 310-322. MR 13, 719.

8. I. Kaplansky, Topics in commutative rings. III, University of Chicago Lecture Notes, Chicago, Ill., 1971. 
9. C. Lanski, Rings with involution whose symmetric elements are regular, Proc. Amer. Math. Soc. 33 (1972), 264-270. MR 45 \#1971.

10. S. Montgomery, Rings with involution in which every trace is nilpotent or regular, Canad. J. Math. 26 (1974).

11. G. Thierrin, Sur les idéaux complètement premiers d'un anneau quelconque, Acad. Roy. Belg. Bull. Cl. Sci. (5) 43 (1957), 124-132.

12. E. T. Wong, Regular rings and integral extension of a regular ring, Proc. Amer. Math. Soc. 33 (1972), 313-315. MR 45 \# 3475.

DEPARTMENT OF MATHEMATICS, CARLETON UNIVERSITY, OTTAWA, ONTARIO, CANADA 